\title{
Avian Diversity in and around Digha, District-East Midnapore (West Bengal, India)
}

\author{
Goutam Patra ${ }^{1}$, Santanu Chakrabarti ${ }^{2}$ \\ ${ }^{1}$ Department of Zoology, Bidhan Nagar Government College, Kolkata, India \\ ${ }^{2}$ Department of Zoology, A.P.C. Roy Government College, Siliguri, India \\ Email: goutampatra.9@gmail.com, scwbes@gmail.com
}

Received 10 March 2014; revised 18 May 2014; accepted 3 June 2014

Copyright @ 2014 by authors and Scientific Research Publishing Inc.

This work is licensed under the Creative Commons Attribution International License (CC BY). http://creativecommons.org/licenses/by/4.0/

c) (i) Open Access

\begin{abstract}
The digha is located at the border of West Bengal and Orissa state. It has both fresh and saline waterbodies rich in aquatic vegetation and several kinds of birds in the harbours in all the seasons. Grassland areas, rice fields, herbs, shrubs and trees located in and around the water bodies provide food and shelter for these birds. Total number of 86 bird species belonging to 10 orders and 35 families were recorded during the 2-year long study period. Passeriformes is the dominant order of birds. But the future of this avian fauna is in danger due to unchecked growth of tourism related hotel industry and urbanisation of the city.
\end{abstract}

\section{Keywords}

Avifauna, Biodiversity, Digha

\section{Introduction}

The Indian subcontinent is very rich in biodiversity. According to an estimate total 1300 bird species found in Indian subcontinent, out of the more than 9000 bird species of the world, over $13 \%$ of the world's bird fauna are found in India [1]-[3]. It is suggested that the avifauna is important for the good health of the ecosystem as these birds play various roles as scavenger, pollinators and predators of insect pests [4] [5]. Digha is a seaside city in the state of West Bengal, India. It is present in East Midnapore district and at the northern end of the Bay of Bengal. The name “Digha”, was referred by Warren Hastings's letters as Brighton of the East [6]. It is located at $21.68^{\circ} \mathrm{N} 87.55^{\circ} \mathrm{E}$. It has an average elevation of 6 metres (22 feet) (Figure 1 and Figure 2). There are three seasons in Digha, mainly summer, monsoon, winter. Average temperature is $28^{\circ} \mathrm{C}-37^{\circ} \mathrm{C}$. It shows great avian diversity 


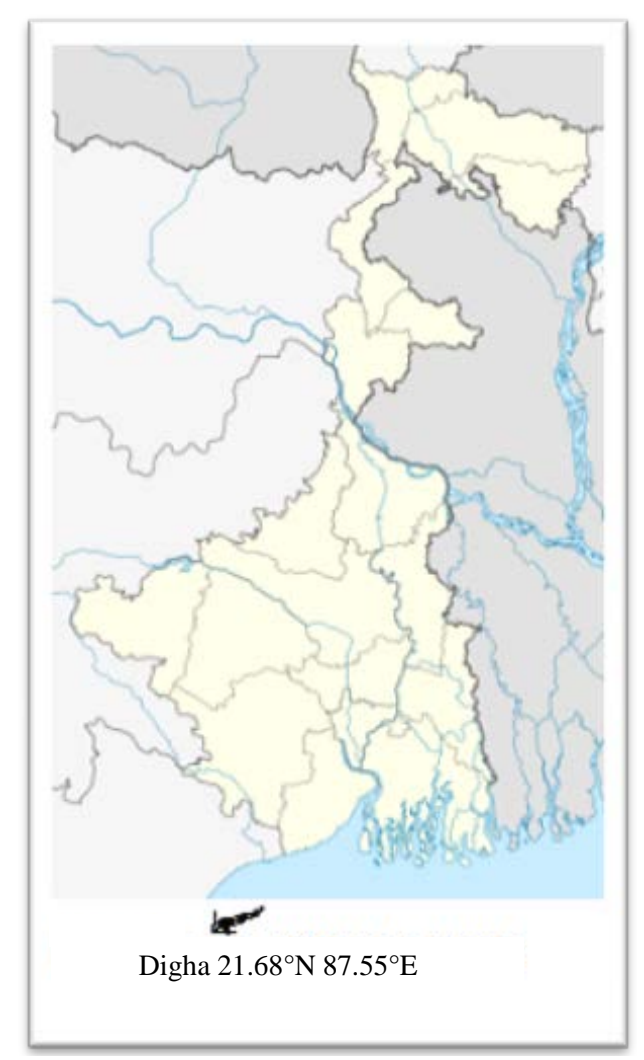

Figure 1. It shows the location of Digha (Photograph taken from http://en.wikipedia.org/wiki).

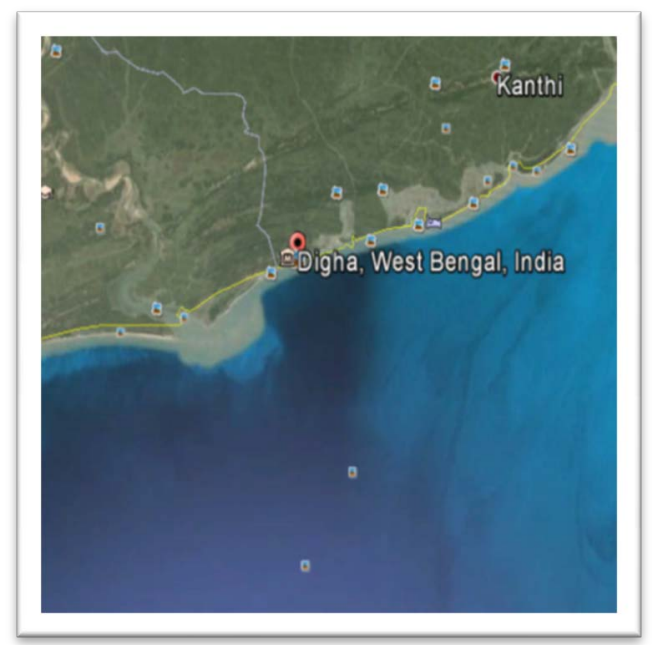

Figure 2. It shows the location of Digha (Photograph taken from www.googleearth.com).

which can attract the attention of bird watchers and ornithologists. The area is surrounded by a large number of small and big ponds, estuaries and Bay of Bengal in southern side of Digha. Nearest areas of Digha are Junput, Shankarpur, Mandarmani and Talsari and these areas also contain a large diversity of avifauna. The present study is not carried out only to prepare the checklist of birds, but to find out their occurrence and to create awareness for their conservation. Of course, it is the need of the hour to conserve the rich diversity of avifauna of Digha. 


\section{Materials and Method}

An extensive study of avifauna was carried out from October 2012 to February 2014 at the regular interval of fifteen days during most active period of birds. The data was recorded in each survey from different habitat types and the observations were carried out with the help of $10-12 \times 50$ Nikon binocular and photography was done with the Cannon SX50 HS and EOS 7D cameras with 150 - 500 mm Sigma lens. Identification of birds was done using field guides book of Grimmett and Selim Ali [1] [7]-[9] and by searching internet resources. Classification of birds was carried out with aid of published references [6] [10]. Picture of study area was taken by using website Google earth and graphs were prepared by using the Microsoft Excel. The avian species are classified on the basis of resident (R), resident migrant (RM) and migrant (M).

\section{Results and Discussion}

The total of 86 species of birds belonging to 10 orders and 35 families were recorded from Digha and its surrounding areas (Table 1 ). This is the first record in Digha avian fauna of West Bengal State which shows quite good avian diversity in this area. Some varieties of birds visit in the winter season for breeding and most of them

Table 1. It shows orders, families, scientific names, common names and habitat of the birds. Here resident $\mathrm{R}=$ resident, RM = resident migrant and $\mathrm{M}=$ migrant.

\begin{tabular}{|c|c|c|c|c|c|}
\hline SL No. & Order & Family & Scientific Name & Common Name & Habit \\
\hline \multirow{35}{*}{1} & \multirow{35}{*}{ Passeriformes } & \multirow{4}{*}{ Picidae } & Micropternus brachyurus & Rofous Woodpecker & $\mathrm{R}$ \\
\hline & & & Dendrocopos macei & Fulvous-breasted Woodpecker & $\mathrm{R}$ \\
\hline & & & Chrysocolaptes lucidus & Greater Golden-back Woodpecker & $\mathrm{R}$ \\
\hline & & & Dinopium benghalense & Lesser Golden-back Woodpecker & $\mathrm{R}$ \\
\hline & & \multirow{4}{*}{ Corvidae } & Dicrurus macrocercus & Black Drongo & $\mathrm{R}$ \\
\hline & & & Corvus splendens & House Crow & $\mathrm{R}$ \\
\hline & & & Corvus macrorhynchous & Jungle Crow & $\mathrm{R}$ \\
\hline & & & Dendrocitta vagabunda & Rufous Treepie & $\mathrm{R}$ \\
\hline & & Alaudidae & Alauda gulgula & Oriental Skylark & $\mathrm{R}$ \\
\hline & & Cisticolidae & Orthotomussutorius & Common Tailorbird & $\mathrm{R}$ \\
\hline & & \multirow{6}{*}{ Passeridae } & Motacilla flava & Yellow-Wagtail & $\mathrm{R}$ \\
\hline & & & Motacilla alba & White-Wagtail & $\mathrm{R}$ \\
\hline & & & Anthus rufulus & Paddyfield Pipit & $\mathrm{R}$ \\
\hline & & & Lonchura punctulata & Scally-Breasted Munia & $\mathrm{R}$ \\
\hline & & & Ploceus philippinus & Baya Weaver & $\mathrm{R}$ \\
\hline & & & Passer domesticus & House Sparrow & $\mathrm{R}$ \\
\hline & & Sylviidae & Turdoides striat & Jungal Babbler & $\mathrm{R}$ \\
\hline & & \multirow{3}{*}{ Muscicapidae } & Zoothera citrina & Orange-Headed Thrush & $\mathrm{R}$ \\
\hline & & & Saxicolodies fulicatus & Indian Robin & $\mathrm{R}$ \\
\hline & & & Saxicola caprata & Pied Bushchat & $\mathrm{R}$ \\
\hline & & \multirow{2}{*}{ Laniidae } & Lanius schach & Rufous-Backed Shrike & RMMMM \\
\hline & & & Lanius excubitor & Great Grey Shrike & $\mathrm{RM}$ \\
\hline & & Pycnonotidae & Pycnonotus cafer & Red-Vented Bulbul & $\mathrm{R}$ \\
\hline & & \multirow{2}{*}{ Nectariniidae } & Nectarinia zeylonica & Purple-Rumped Sunbird & $\mathrm{R}$ \\
\hline & & & Cinnyris asiaticus & Purple Sunbird & $\mathrm{R}$ \\
\hline & & \multirow{2}{*}{ Oriolidae } & Oriolus kundoo & Indian Golden Oriole & $\mathrm{R}$ \\
\hline & & & Oriolus xanthornus & Black-Hooded Oriole & $\mathrm{R}$ \\
\hline & & \multirow{4}{*}{ Sturnidae } & Sturnia malabrica & Chestnut-Tailed Starling & $\mathrm{R}$ \\
\hline & & & Acridotheres fuscus & Jungle Myna & $\mathrm{R}$ \\
\hline & & & Acridotheres tristis & Common Myna & $\mathrm{R}$ \\
\hline & & & Sturnus contra & Asian Pied Starling & $\mathrm{R}$ \\
\hline & & \multirow{3}{*}{ Megalaimidae } & Megalaima haemacephala & Coppersmith Barbet & $\mathrm{R}$ \\
\hline & & & Megalaima asiatica & Blue-Throated Barbet & $\mathrm{R}$ \\
\hline & & & Megalaima lineata & Lineated Barbet & $\mathrm{R}$ \\
\hline & & Rhipiduridae & Rhipidura albicollis & White-Throated Fantail & $\mathrm{R}$ \\
\hline
\end{tabular}




\section{Continued}

\begin{tabular}{|c|c|c|c|c|c|}
\hline & & Zosteropidae & Zosterops palpebrosus & Oriental White-Eye & $\mathrm{R}$ \\
\hline \multirow{2}{*}{2} & \multirow{2}{*}{ Cuculiformess } & \multirow{2}{*}{ Cuculidae } & Eudynamys scolopaceus & Asian Koel & $\mathrm{R}$ \\
\hline & & & Centropus sinensis & Greater Coucal & $\mathrm{R}$ \\
\hline \multirow{19}{*}{3} & \multirow{19}{*}{ Ciconiiformes } & \multirow{5}{*}{ Charadriidae } & Vanellus malabaricus & Yellow-Wattled Lapwing & RM \\
\hline & & & Vanellus indicus & Red-Wattled Lapwing & $\mathrm{RM}$ \\
\hline & & & Tringa gareola & Sand Piper & M \\
\hline & & & Himantopus himantopus & Black Winged Stilt & $\mathrm{RM}$ \\
\hline & & & Charadrius dubius & Little Ringed Plover & $\mathrm{R}$ \\
\hline & & \multirow{5}{*}{ Ardeidae } & Bubulcus ibis & Cattle Egret & $\mathrm{R}$ \\
\hline & & & Egretta garzetta & Little Egret & $\mathrm{RM}$ \\
\hline & & & Casmerodius albus & Large Egret & $\mathrm{RM}$ \\
\hline & & & Ardeola grayii & Indian Pond Heron & $\mathrm{R}$ \\
\hline & & & Mesophoyx intermedia & Median Egret & $\mathrm{RM}$ \\
\hline & & Phalacrocoracidae & Phalacrocorax niger & Little Cormorant & $\mathrm{R}$ \\
\hline & & \multirow{2}{*}{ Jacanidae } & Hydrophasianus chirurgus & Pheasant-Tailed Jacana & $\mathrm{R}$ \\
\hline & & & Metopidius indicus & Bronze-Winged Jacana & $\mathrm{R}$ \\
\hline & & Anhingidae & Anhinga melanogaster & Darter or Snake Bird & M \\
\hline & & \multirow{2}{*}{ Accipitridae } & Anastomus oscitans & Asian Openbill-Stork & $\mathrm{RM}$ \\
\hline & & & Aquila spe. & Eagle & RM \\
\hline & & \multirow{3}{*}{ Ciconiidae } & Elanus caeruleus & Black Shouldered Kite & $\mathrm{R}$ \\
\hline & & & Milvus migrans & Black Kite & $\mathrm{R}$ \\
\hline & & & Haliastur indus & Brahminy Kite & $\mathrm{R}$ \\
\hline \multirow{8}{*}{4} & \multirow{8}{*}{ Coraciformes } & \multirow{4}{*}{ Alcedinidae } & Ceryle rudis & Lesser Pied Kingfisher & $\mathrm{RM}$ \\
\hline & & & Pelargopsis capnsis & Strok-Billed Kingfisher & $\mathrm{R}$ \\
\hline & & & Alcedo atthis & Small Blue Kingfisher & $\mathrm{R}$ \\
\hline & & & Halcyon smyrnensis & White-Breasted Kingfisher & $\mathrm{R}$ \\
\hline & & \multirow{3}{*}{ Meropidae } & Merops orientalis & Small Bee-Eater & $\mathrm{R}$ \\
\hline & & & Merops philippinus & Blue-Tailed Bee Eater & $\mathrm{RM}$ \\
\hline & & & Merops leschenaulti & Chestnut-Headed Bee-eater & $\mathrm{R}$ \\
\hline & & Coraciidae & Coracias benghalensis & Indian Roller & $\mathrm{RM}$ \\
\hline \multirow{6}{*}{5} & \multirow{6}{*}{ Columbiformes } & \multirow{6}{*}{ Columbidae } & Streptopelia chinensis & Spotted Dove & $\mathrm{R}$ \\
\hline & & & Streptopelia tranquebarica & Red Collard-Dove & $\mathrm{R}$ \\
\hline & & & Columba livia & Blue Rock Pigeon & $\mathrm{R}$ \\
\hline & & & Streptopelia chinensis & Spotted Dove & $\mathrm{R}$ \\
\hline & & & Streptopelia decaoto & Eurasian Collared Dove & $\mathrm{R}$ \\
\hline & & & Treron phoenicopterus & Yellow-Footed Green Pigeon & $\mathrm{RM}$ \\
\hline 6 & Gruiformes & Rallidae & Amaurornis phoenicurus & White-Breasted Warehen & $\mathrm{R}$ \\
\hline \multirow{2}{*}{7} & \multirow{2}{*}{ Psittaciformes } & \multirow{2}{*}{ Psittacidae } & Psittacula krameri & Rose-Ringed Parakeet & $\mathrm{R}$ \\
\hline & & & Psittacula eupatria & Alexanderine Parakeet & $\mathrm{R}$ \\
\hline \multirow{9}{*}{8} & & & Glareola lactea & Small Pratincole & $\mathrm{RM}$ \\
\hline & & Clareolidao & Ichthyaetus ichthyaetus & Palla’s Gull & M \\
\hline & & Glareomade & Chroicocephalus brunnicephalus & Black-Headed Gull & M \\
\hline & & & Chroicocephalus ridibundus & Brown-Headed Gull & M \\
\hline & Charadriniiformes & Starnidae & Gelochelidon nilotica & Gull-Billed Tern & M \\
\hline & & steminude & Sterna aurantia & River Tern & $\mathrm{R}$ \\
\hline & & Scolonacidas & Gallinago gallinago & Common Snipe & M \\
\hline & & & Tringa glareola & Wood Sandpiper & M \\
\hline & & Recurvirostridae & Himantopus himantopus & Black-Winged Stilt & M \\
\hline 9 & Galliformes & Phasianidae & Gallus gallus & Red Jungle fowl & $\mathrm{R}$ \\
\hline 10 & Strigiformes & Strigidae & Otus lettia & Collared Scopes Owl & $\mathrm{R}$ \\
\hline & & & Glaucidium brodiei & Collared Owlet & $\mathrm{R}$ \\
\hline
\end{tabular}


permanently reside in this habitat due to presence of ample amount of avian food in and around Digha. Most of the avian species are resident (R), resident migrant (RM) and very few are migrant (M).

Coastal areas of Digha exhibit significant quantitative variation in avifauna. Among 10 orders of recorded birds Passeriformes consists of 42\% (Figure 3) of total families out of 35 (Table 1), and it includes Picidae, Corvidae, Alaudidae, Cisticolidae, Passeridae, Sylviidae, Muscicapidae Laniidae Pycnonotidae, Nectariniidae, Oriolidae, Sturnidae, Megalaimidae, Rhipiduridae and Zosteropidae. Birds belonging to orders like Gruiformes and Galliformes constitute 1\% of birds of total species surveyed (Figure 3). Passeriformes consists of 42.86\%, and Cuculiformes, Psittaciformes, Gruiformes and Galliformes, Strigiformes, Columbiformes consists of 2.86\% order each of total bird's species (Figure 4).

Avifauna belonging to families Columbidae and Passeridae constitute $6.97 \%$ of the total bird species. Pycnonotidae, Rhipiduridae, Zosteropidae, Aludidae, Cisticolidae Sylviidae, Phalacrocoracidae, Anhingidae, Coraciidae, Rallidae, Recurvirostridae, and Phasianidae constitute 1.16\% each of the total bird species (Figure 5). Further it was interesting to note that passerine birds dominated the diversity with 36 species as compared to non passerine (50 species) (Table 1). Family wise analysis showed that family Columbidae and Passeridae (6 species each) dominated the avian fauna followed by Charadriidae, Ardeidae (5 species each), Picidae, Corvidae, Sturnidae, Alccedinidae, Glareolidae (4 species each), Muscicapidae, Megalaimidae, Ciconiidae, Meropidae (3 species each), Laniidae, Nectariniidae, Orilidae, Cuculidae, Jacanidae, Accipitridae, Psittacidae, Sternidae, Scolopacidae, Strigidae (2 species each), Pycnonotidae, Rhipiduridae, Zosteropidae, Sylviidae, Phalacrocoracidae, Anhingidae, Coraciidae, Rallidae, Recurvirostridae, and Phasianidae Aludidae, Cisticolidae (1 species each)
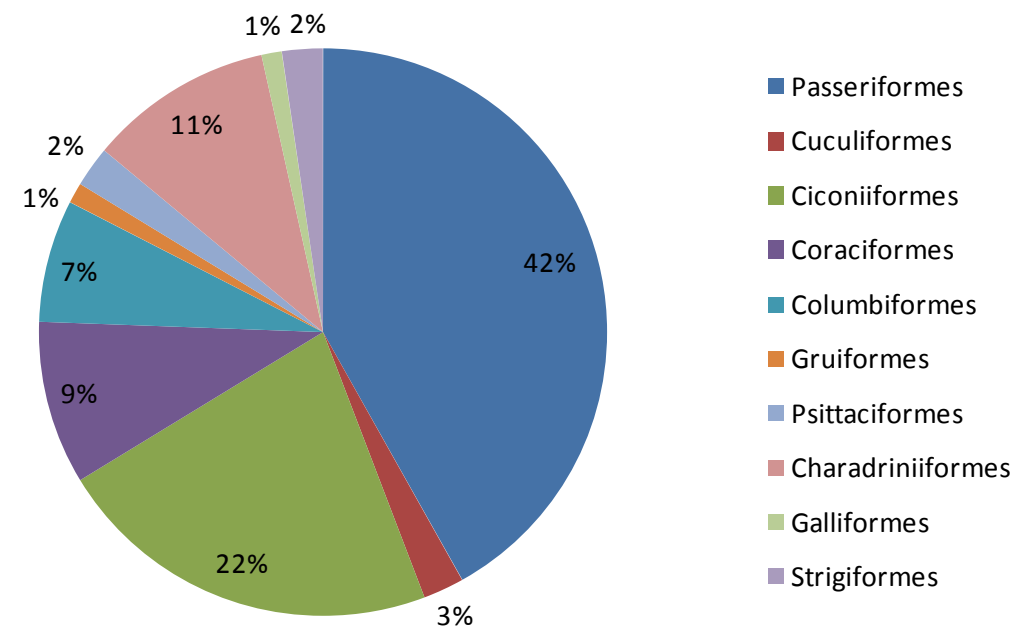

Figure 3. It shows percentages (\%) of Species in different families of avifauna.

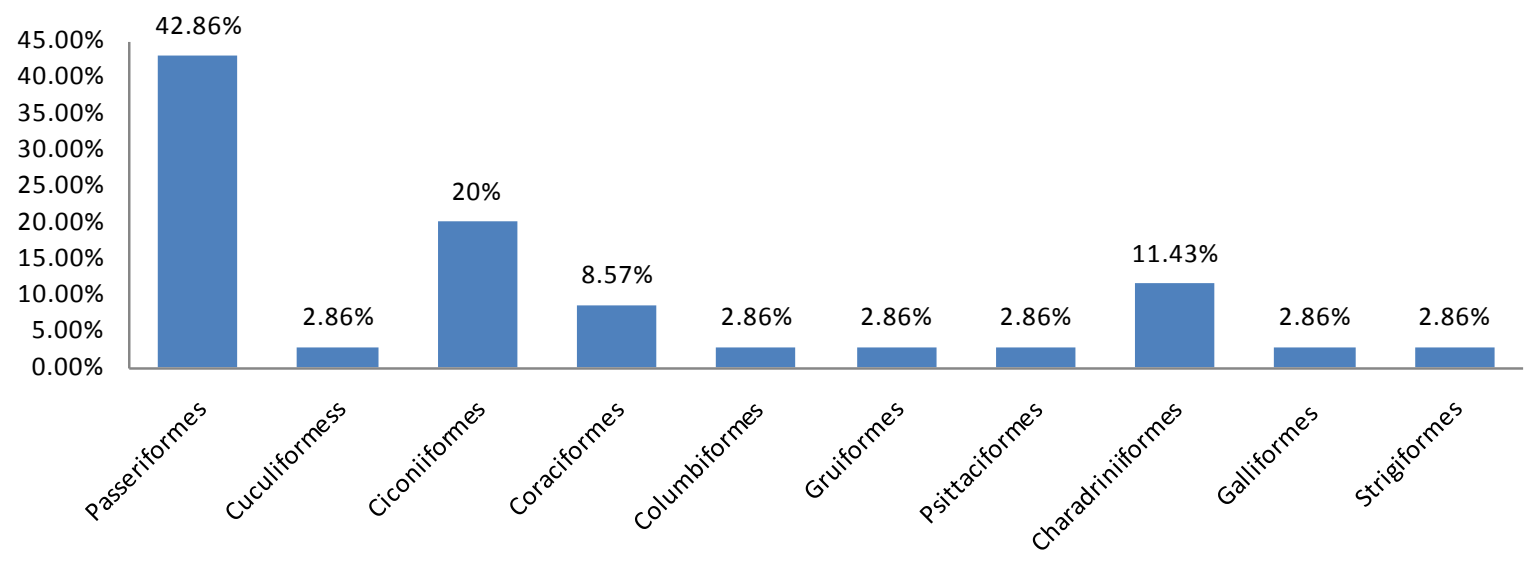




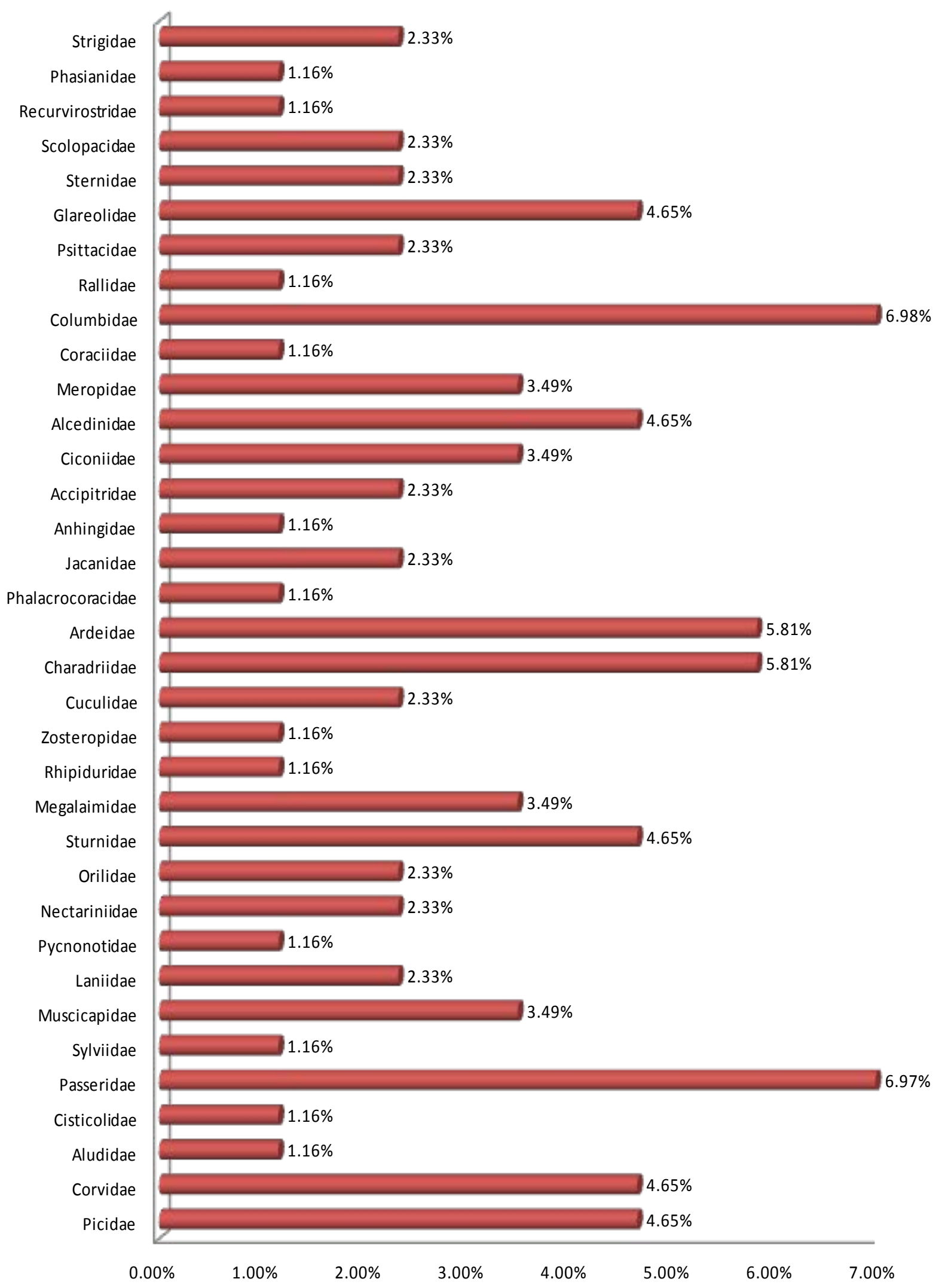

Figure 5. It shows the family wise percentages of avian fauna. 


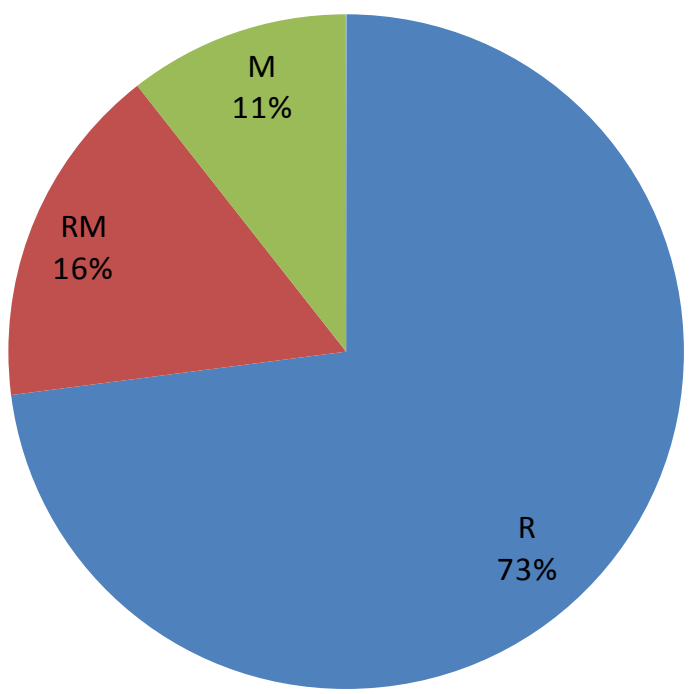

Figure 6. It shows percentages (\%) of resident (R), resident-migrant (RM) and migrant (M) avifauna.

were poorly represented in the area (Table 1). Analysis of the data on residential status revealed that out of 86 species, $73 \%$ were resident, $16 \%$ resident migrant (RM) and $11 \%$ are migrant (M) (Figure 6).

\section{Conclusion}

The present study establishes the richness of the Digha with respect to bird fauna which is an excellent indicator of ecological health. However, the future of the avian fauna of Digha is under threat from unabated urbanisation, increased bird hunting, and pouring of hazardous agricultural chemicals into the water bodies affecting the fish eating birds and others (migratory birds mainly). Pollution and disturbances related to water sports and overcrowding of tourist are also expected to severely disturb the ecological balance (specially the avian diversity) of this area.

\section{References}

[1] Grimmett, R., Inskipp, C. and Inskipp, T. (1998) Pocket Guide to the Birds of Indian Subcontinent. Oxford University Press, Mumbai.

[2] Sharma, I.K. (1982) Adverse Effects of Air, Water and Soil Pollutions on Flora and Fauna of Towns and Villages of Western Rajasthan. Symposium on Environment Consciousness, Problems of Pollution and Conservation in Rajasthan, 1-3 October 1982.

[3] Zoological Survey of India, Contribution to the Faunal Diversity of India, Aves.

[4] Padmavati, A., Alexandar, R. and Anbarashan, M. (2010) Our Nature. 8, 247-253.

[5] Bhattacharjee, P.C. and Hazarika, B.C. (1985) Roosting Sites and Roosting Birds at Gauhati Muncipal Area. Second Intenational Symposium on Life Sciences, 14-16 November 1985, NEHU Shillong.

[6] http://en.wikipedia.org/wiki/Digha

[7] Ali, S. (2012) The Books of Indian Birds. 13th Edition, Oxford University Press, Delhi.

[8] Ali, S. and Ripely, S.D. (1987) Compact Handbook of Birds of India and Pakistan. Oxford University Press, Bombay.

[9] Balachandran, S., et al. (2009) Bird Atlas of Chilka. Bombay Natural History Socity, India.

[10] http://data.gbif.org/search 
Scientific Research Publishing (SCIRP) is one of the largest Open Access journal publishers. It is currently publishing more than 200 open access, online, peer-reviewed journals covering a wide range of academic disciplines. SCIRP serves the worldwide academic communities and contributes to the progress and application of science with its publication.

Other selected journals from SCIRP are listed as below. Submit your manuscript to us via either submit@scirp.org or Online Submission Portal.
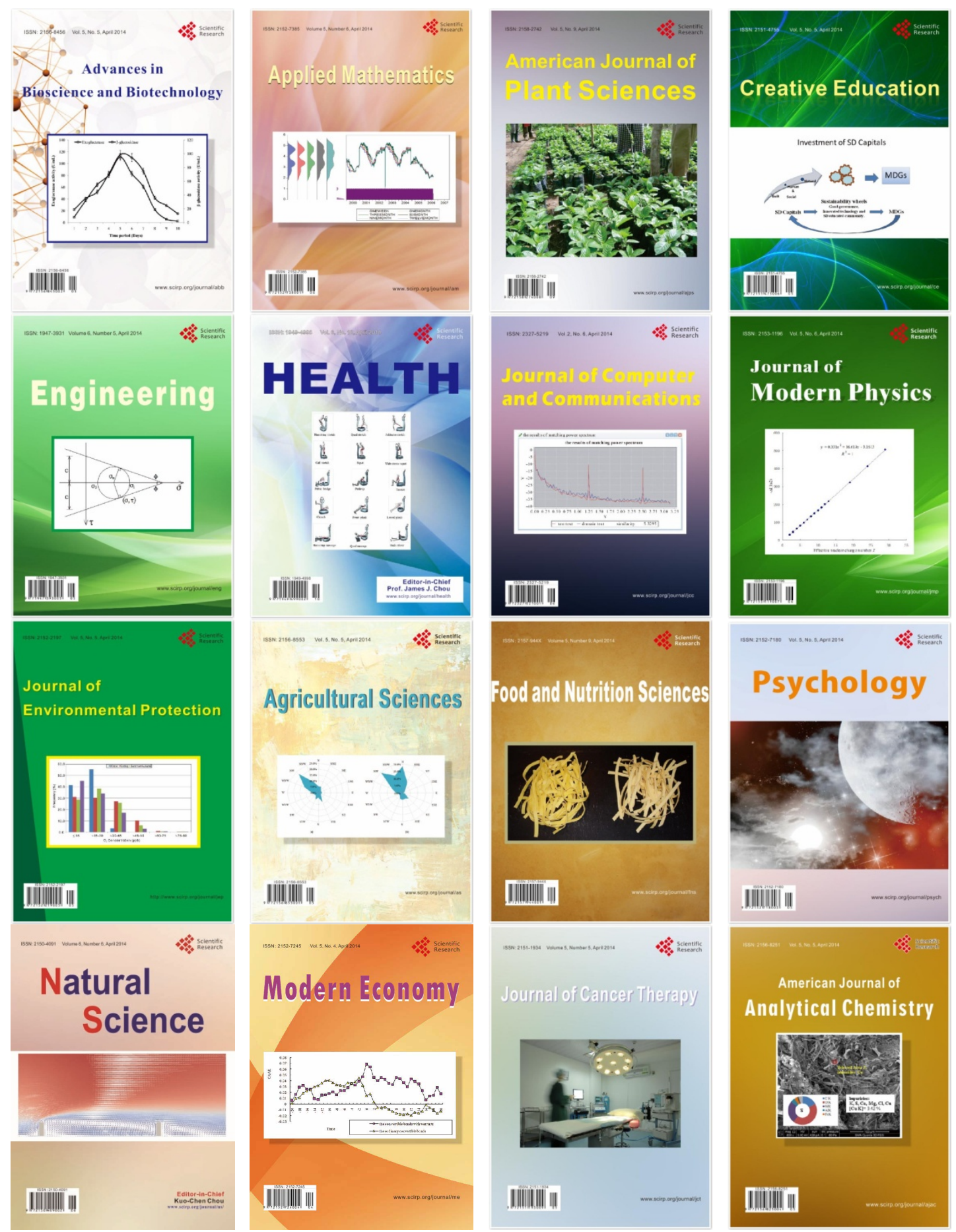